\title{
Cooperative Function of Tbx1 and Brn4 in the Periotic Mesenchyme is Necessary for Cochlea Formation
}

\author{
Evan M. Braunstein ${ }^{1}$, E. Bryan Crenshaw III ${ }^{2,3}$, Bernice E. Morrow ${ }^{1}$, and Joe C. Adams ${ }^{4}$ \\ ${ }^{1}$ Department of Molecular Genetics, Albert Einstein College of Medicine, 1300 Morris Park Avenue, Bronx, NY 10461, USA \\ ${ }^{2}$ Mammalian Neurogenetics Group, Center for Childhood Communication, The Children's Hospital of Philadelphia, \\ 34th and Civic Center Boulevard, Philadelphia, Philadelphia, PA 19104, USA \\ ${ }^{3}$ Department of Otorhinolaryngology: Head and Neck Surgery, University of Pennsylvania Medical School, Philadelphia, \\ PA 19104, USA \\ ${ }^{4}$ Department of Otolaryngology, MA Eye and Ear Infirmary, 243 Charles Street, Boston, MA 02114, USA
}

Received: 30 October 2007; Accepted: 3 January 2008; Online publication: 30 January 2008

\begin{abstract}
The T-box transcription factor TBX1 has been identified as the major gene responsible for the etiology of velocardiofacial syndrome/DiGeorge syndrome (VCFS/DGS). Conductive hearing loss occurs in a majority of patients with this syndrome, while sensorineural deafness has also been reported in some cases. Mutations in POU3F4/BRN4, a POU domain transcription factor, cause DFN3, an X-linked nonsyndromic form of deafness characterized by mixed conductive and sensorineural hearing loss. Inactivation of the murine orthologues of these genes causes similar defects to those seen in humans and has provided excellent models for the study of inner ear development. Tbx1 and Brn4 are expressed in the mesenchymal cells surrounding the otic vesicle and have been shown to play roles in cochlear outgrowth. Furthermore, expression of $B r n 4$ is reduced in $T b x 1$ null mutants, suggesting a possible genetic interaction between these genes. To test whether Tbx1 and Brn4 function in a common pathway, mice mutant for both genes were generated and analyzed for inner ear defects. Brn4-;Tbx1+/- mutants displayed a significant reduction in the number of turns of the cochlea compared to Brn4- or Tbx1+/- mice. In addition, Brn4-;Tbx1+/- mice displayed structural defects in
\end{abstract}

Correspondence to: Bernice E. Morrow - Department of Molecular Genetics - Albert Einstein College of Medicine 1300 Morris Park Avenue, Bronx, NY 10461, USA. Telephone: +1-718-4304274; fax: +1718-4308778; email: morrow@aecom.yu.edu the apical cochlea indicative of Mondini dysplasia found in patients with either VCFS/DGS or DFN3. These data establish a genetic interaction between $T b x 1$ and Brn 4 relevant to human disease and indicate a function of these genes in signaling from the periotic mesenchyme to the otic vesicle to direct proper coiling of the cochlear duct.

Keywords: inner ear, genetic interaction, Mondini dysplasia

\section{INTRODUCTION}

Approximately 1 in 1,000 infants suffers from severe or profound hearing loss, sometimes due to abnormal development of the ear. Two genes that have been identified as having prominent roles in ear morphogenesis are TBX1 and POU3F4/BRN4. TBX1 is a member of the T-box family of transcription factor genes. Deletion or mutation of $T B X 1$ is associated with velocardiofacial syndrome/DiGeorge syndrome (VCFS/DGS) and is characterized by defects in the development of structures derived from the pharyngeal apparatus. Conductive hearing loss associated with chronic otitis media occurs in a majority of patients, while sensorineural deafness has also been reported in approximately $10 \%$ of cases (Digilio et al. 1999; Ford et al. 2000; Reyes et al. 1999). Pathological analysis performed on hearing impaired patients revealed the presence of Mondini dysplasia, character- 
ized by a reduced number of cochlear turns and an incomplete interscalar septum (Holden and Linthicum 2005; Ohtani and Schuknecht 1984).

$B R N 4 / P O U 3 F 4$ encodes a member of the POU domain family of transcription factors. Mutations in $B R N 4$ cause a nonsyndromic form of deafness known as DFN3, classified by its X-linked mode of inheritance (de Kok et al. 1995). Afflicted individuals are characterized clinically by mixed conductive and sensorineural hearing loss and pathologically by defects in the bony labyrinth of the inner ear. These abnormalities include Mondini dysplasia, cochlear hypoplasia, and stapes fixation (Arellano et al. 2000; Phelps et al. 1991). Mondini dysplasia is a common radiological finding in cases of idiopathic congenital hearing loss (Zheng et al. 2002), and elucidating its molecular basis will aid in our understanding of the causes of deafness with unknown etiologies.

Mouse models have made it possible to study the molecular origins of the ear pathology found in both VCFS/DGS and DFN3. Mice homozygous null for Tbx 1 die at birth and exhibit severe ear defects due to early arrest of inner ear development (Raft et al. 2004; Vitelli et al. 2003). The otic vesicle fails to undergo normal morphogenesis, resulting in a cystic otic vesicle with a missing cochlea and vestibular system. In addition, the cochleovestibular ganglion, which derives from the otic vesicle, is duplicated due to ectopic activation of neurogenesis in cells lacking Tbx1 (Raft et al. 2004). This suggests that Tbx1 acts as a selector gene to control sensory organ and neural fate specification in the otic vesicle. $T b x 1$ is expressed in both the otic vesicle and surrounding periotic mesenchyme, and conditional ablation of Tbx 1 in the otic vesicle produced an inner ear phenotype identical to Tbx $1-/-$ embryos. This indicated that $T b x 1$ expression in the otic vesicle controls early inner ear patterning and morphogenesis tissue autonomously (Arnold et al. 2006a). However, recently the expression domain of $T b \times 1$ in the periotic mesenchyme was shown to be necessary for cochlear outgrowth, supporting a role for $T b x 1$ in mesenchymal signaling to the otic vesicle (Xu et al. 2007a, b).

Inactivation of $\mathrm{Brm} 4$ in the mouse also produces inner ear abnormalities. Brn 4 is not expressed in the otic vesicle but is expressed throughout the periotic mesenchyme (Phippard et al. 1998). Mice null for $\mathrm{Brn} 4$ exhibit profound hearing loss due to a reduction in endocochlear potential (Minowa et al. 1999). This is caused by defects in spiral ligament fibrocytes, which are involved in the potassium homeostasis of the endolymph. Additionally, some Brn4 null mice display a reduction in cochlear coiling (Phippard et al. 1999). The normal mouse cochlea consists of 1.75 turns; however, approximately $25 \%$ of $\mathrm{Brm} 4$ null mice have less than 1.5 turns, indicating a role for $\mathrm{Brn} 4$ in epithelial-mesenchymal interactions during ear development. This further suggests that signaling from the periotic mesenchyme to the otic vesicle is necessary for cochlear outgrowth.

Recently, we found that Brm4 expression in the periotic mesenchyme is reduced in Tbx $1-/-$ embryos but is unaffected in otic vesicle-specific conditional mutants of $T b x 1$, suggesting that $T b x 1$ in the periotic mesenchyme may regulate $\mathrm{Brn} 4$ expression or proliferation of Brn4-expressing cells (Arnold et al. 2006a). To test whether these genes function in a common pathway, mice mutant for both Tbx1 and Brm4 were examined for cochlear defects. The basal portion of the cochlea was abnormal in both Brn4- and Brn4-; Tbx1+/- mice, however, double mutant mice displayed defects in the apical portion of the cochlea as well. In addition, only $15 \%$ of $\mathrm{Brn} 4-$ mice failed to complete 1.75 turns of the cochlea, while Brn4-;Tbx1+/- mice displayed a fully penetrant reduction to 1 or 1.5 cochlear turns. These experiments support the roles of Tbxl and Brn4 in mesenchymal signaling necessary for proper inner ear formation. Furthermore, they demonstrate a genetic interaction likely involved in the etiology of congenital hearing loss associated with Mondini dysplasia.

\section{MATERIALS AND METHODS}

\section{Experimental animals}

The generation the $T b x 1$ null allele has been previously described (Arnold et al. 2006b). Brm4 null mice were obtained from Dr. E. Bryan Crenshaw (Ahn and Crenshaw, unpublished data). The $\mathrm{Brn} 4$ null allele was generated by replacing the entire $\mathrm{Brn} 4 \mathrm{ORF}$ with a Cre transgene using the same method as previously described (Phippard et al. 1999). Brn4 hemizygous males or heterozygous females in a mixed genetic background were crossed to $T b x 1+/-$ mice maintained on a C57BL/ 6 background. Mice were genotyped with the following primers: Tbx 1 null $5^{\prime}$-atgtaaaagcgtcct ggcca-3', 5'-cggagctggtgtgtacata-3'; Brn4 null 5'caatgctgtttcactggttatg-3', $5^{\prime}$-cattgcccetgtttcactatc- $3^{\prime}$. Male embryos were identified by PCR genotyping with primers to the Sry gene 5'-cagccctacagccacatgat- $3^{\prime}, 5^{\prime}$ gagtacaggtgtgcagctcta- $3^{\prime}$. All animals were maintained on a 24-h dark/light cycle; the day of the plug was recorded as E0.5. Embryos were staged using a mouse anatomical atlas, and littermates were used as controls in all experiments.

\section{Histology}

For histopathological analysis of adult mice, animals were sacrificed and their ears perfused with $10 \%$ formalin and $1 \%$ acetic acid, decalcified in EDTA, 
embedded in paraffin, sectioned and stained with hematoxylin and eosin.

\section{Immunohistochemistry}

Embryos were isolated in cold PBS followed by fixation in $4 \%$ PFA overnight at $4^{\circ} \mathrm{C}$, ethanol dehydration and embedded in paraffin wax. Seven micrometer thick sections were treated with either polyclonal rabbit antiTbx1 serum (Zymed) 1:500, affinity-purified rabbit polyclonal anti-Brn4 Ab (Phippard et al. 1998) 1:400, anti-cleaved Caspase 3 (R\&D Systems) 1:1,000, or antiphospho-Histone 3 (Upstate) 1:200 in TBS/0.1\% Triton X-100/5\% goat serum/2\%BSA. Sections were incubated for $1 \mathrm{~h}$ at room temperature and visualized with a biotinylated goat anti-rabbit IgG conjugate (1:200; DakoCytomation), avidin-biotin complex/HRP formation (DakoCytomation) and $\mathrm{DAB} /$ chromogen reaction (DakoCytomation). For immunostaining of adult tissue, slides were dewaxed in xylenes, hydrated, and blocked for nonspecific antibody binding with $5 \%$ normal horse serum for $30 \mathrm{~min}$. Primary antibodies were applied and sections were incubated therein overnight at room temperature in a humid chamber. Biotinylated secondary antibodies (Jackson Immunoresearch, West Grove, PA, USA) applied for $1 \mathrm{~h}$, followed by avidin-biotin-horseradish peroxidase reagent (ABC kit, Vector Laboratories, Burlingame, CA, USA) and then hydrogen peroxide/diaminobenzidine to form the reaction product. In some cases, diaminobenzidine was replaced by VIP reagent (Vector Labs) or a mixture of diaminobenzidine and VIP to produce a reaction product that would not be confused with melanin pigment in the tissue. Primary antibodies included rabbit anti-aquaporin1 (Chemicon, http://www.chemicon.com), mouse anti-Na ${ }^{+}$, $\mathrm{K}^{+}$-ATPase (Developmental Studies Hybridoma Bank) and rabbit anti-connexin 26 (Zymed, http:// www.invitrogen.com).

\section{RESULTS}

Reduced number of cochlear turns in Brn4-;Tbx1+/- mice

During ear development, the cochlear duct forms as a canal that extends ventrally from the otic vesicle. Interactions between the growing epithelium and surrounding periotic mesenchyme are thought to be necessary for proper inner ear formation (Chang et al. 2002; Pirvola et al. 2004). Despite X-linked mosaicism, $\mathrm{Brm} 4$ heterozygous null mice have normal inner ears, while $25 \%$ of Brn4 homozygous null mice were reported to have a significant reduction in the number of cochlear turns (Phippard et al. 1999). Although incomplete in penetrance, this defect suggests that $\mathrm{Brm} 4$ functions in mesenchymal signaling to the epithelium. Furthermore, while $T b x 1+/-$ mice have normal inner ears, the periotic mesenchyme domain of $T b x 1$ is necessary for cochlear outgrowth (Liao et al. 2004; Xu et al. 2007a). To investigate the cooperative roles of $\mathrm{Brm} 4$ and $T b x 1$ in mesenchymal-epithelial interactions, mice with null alleles for both $T b x 1$ and $B m 4$ were generated and analyzed for inner ear defects.

We first examined cochlear length in 3-month old male mice by histological methods. To accurately assess cochlear growth, cochlear length was evaluated in half turns. Transverse sections through the modiolus of a Tbx $1+/-$ cochlea revealed four half turns, identical to a wild type cochlea (Fig. 1A). Analysis of both Brm4- and Brn4-;Tbx1+/- mice revealed defects in cochlear coiling. Out of $10 \mathrm{Brn} 4-$ mice examined bilaterally, eight formed four half turns of the cochlea (Fig. 1B). One mouse exhibited only three half turns bilaterally, while another formed three half turns in one ear and four turns in the other (Table 1). In contrast, all 10 Brn4-;Tbx1+/- mice examined displayed a bilateral reduction in cochlear coiling to two or three half turns, indicating an early arrest in cochlear growth (Table 1 ). Furthermore, the organ of Corti in Brn4-; Tbx 1+/mice was either absent or abnormally present in the apical cochlea. For example, in one case the developing cochlea twisted $180^{\circ}$ as it grew to become the second turn so that the organ of Corti is present in reverse orientation. This caused the normally apical surface of the sensory organ to be directed toward the base of the cochlea (Fig. 1D). In another case, the epithelium of the second turn grew abnormally so that the pitch of half turn three is about $45^{\circ}$ basal to its normal position (Fig. 1E). Both of these cases exhibited truncated cochleas, and the abnormal positioning of the organ of Corti is likely secondary to the aberrant growth, misguidance or termination of the cochlear duct. The cochlea of the most severely affected Brn4-; Tbx $1+/-$ mice $(\sim 25 \%)$ failed to extend as far as a third half turn and instead terminated abruptly after the second half turn (Fig. 1F). These data indicated a genetic interaction between $T b x 1$ and $B r m 4$ and suggest that these genes are involved in mesenchymal-epithelial interactions that direct cochlear coiling.

\section{Defects in mesenchymal-derived structures of the cochlea}

Histological analysis of Brn4- and Brn4-;Tbx1+/mice revealed defects in the structural components of the cochlea. Sensory cells that border the endolymphatic space are epithelial and are derived from the otic vesicle. Most cells that do not directly border the endolymphatic space are mesenchymal in origin. These cells were found to be abnormal in the basal turn of the cochlea of both Brn4- and Brn4-; Tbx1+/- 

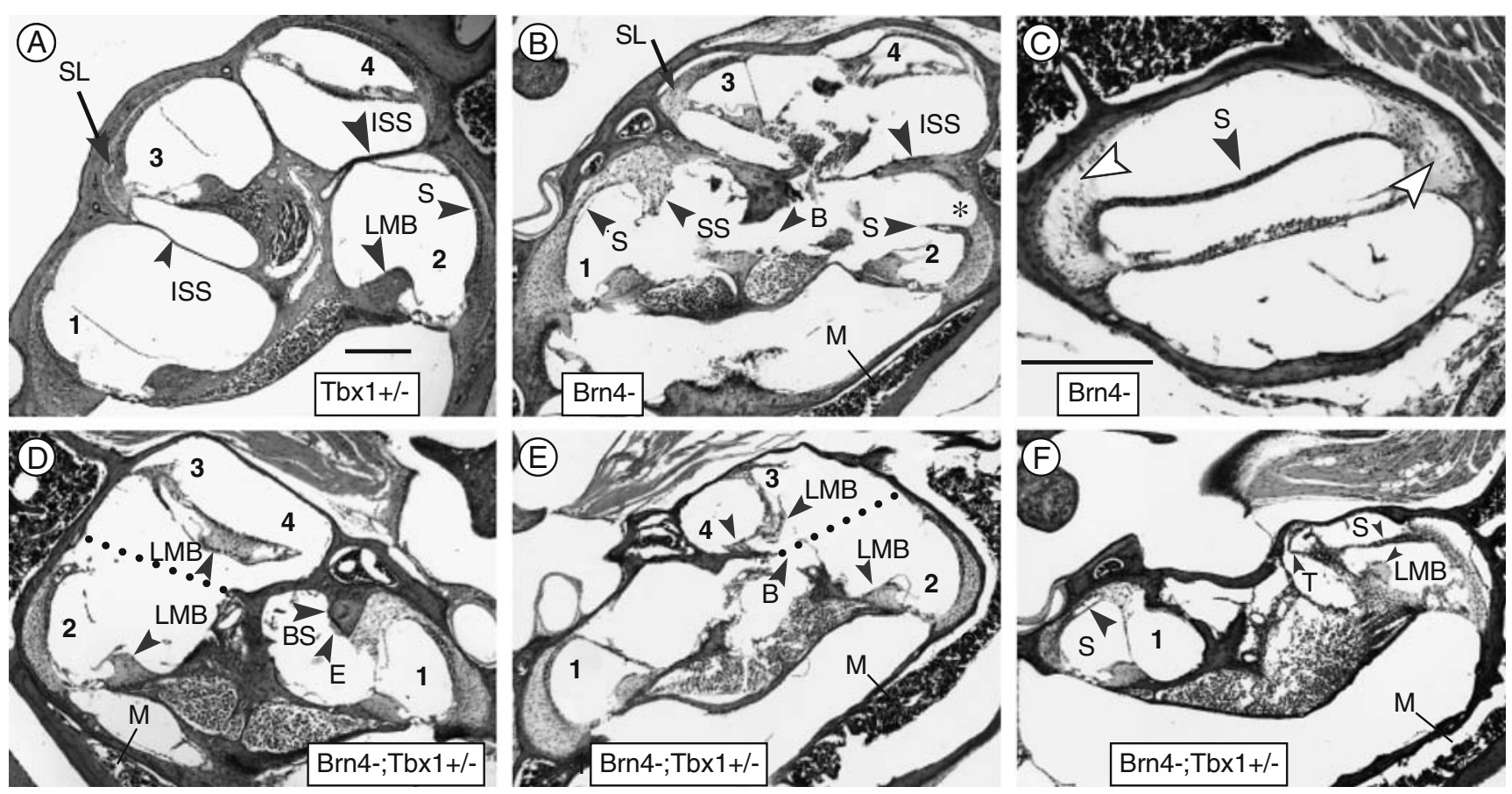

labeled (4). E Left ear of a Brn4-;Tbx1+/- mouse exhibiting an organ of Corti in the second turn oriented at right angles to its normal position. At (3), the basilar membrane is attached to the lateral wall but there is no spiral ligament present. The cochlear duct is shortened, with its apicalmost extremity noted by the arrowhead (4). F Left ear of a Brn4-;Tbx1+/mouse that developed only a basal turn of the cochlea. The stria vascularis in the lower basal turn (1) contains a cyst (arrowhead) and the suprastrial region is hypertrophied. The spiral ligament of the upper basal turn is poorly developed and is barely attached to the stria vascularis, which forms the apical wall of the scala media. The apical cochlear duct is tightly coiled and terminates near the arrowhead $(T)$. $B$, missing bone; $B S$, bone spur; $E$, eosinophillic substance attached to bone spur; ISS, interscalar septum; $L M B$, spiral limbus; $M$, marrow; $S L$, spiral ligament; $S$, stria vascularis; $S S$, suprastrial region; and $T$, termination of cochlear duct. An asterisk marks detachment of the stria from the spiral ligament. Scale bar in $\boldsymbol{A}$ is $100 \mu \mathrm{m}$ and applies to all panels except $\mathbf{C}$. The scale bar in $\boldsymbol{C}$ indicates $100 \mu \mathrm{m}$.

mice, indicating that these defects are due to the absence of the Brn4 gene. However, Brn4-;Tbx1+/mice displayed additional defects in the apical cochlea, again supporting a genetic interaction between Tbx 1 and Brn4.

Two mesenchymal defects were consistently observed in Brn4-; Tbx 1+/- mice that were not found in Brn4- mice. In normal ears, there is a bony interscalar septum that separates the lower turns from the upper turns (Fig 1A). In Brn4- mice, this septum was

\section{TABLE 1}

Cochlear length of adult Brn4- and Brn4-;Tbx1+/- mice

\begin{tabular}{lllll}
\hline & \multicolumn{4}{c}{ Number of half turns $^{a}$} \\
\cline { 2 - 4 } \multicolumn{1}{c}{ Genotype } & \multicolumn{2}{c}{4} & $<4$ & $<3$ \\
\hline Brn4- $(n=20)$ & $17(85 \%)$ & $1(5 \%)$ & $2(10 \%)$ & 0 \\
Brn4-;Tbx1+/- $(n=20)$ & 0 & $7(35 \%)$ & $8(40 \%)$ & $5(25 \%)$ \\
\hline
\end{tabular}

Ten mice were analyzed bilaterally for each genotype for a total of 20 cochleas.

${ }^{a}$ Defined as follows: 4 four complete half turns present, $<4$ truncated upper second turn, $<3$ truncated lower second turn, 2 basal turn present only 
abnormally thick and sometimes incomplete, but it was always present (Fig. 1B). In Brn4-; Tbx+/- mice, the interscalar septum was missing over the more apical half turn of the base (dotted lines in Fig. 1D, E). Additionally, the cross-sectional area of the spiral ligament gradually decreases in size from base to apex in wild type mice. Brn4- mice displayed a relatively normal spiral ligament in the second turn (Fig. 1B); however, in Brn4-;Tbx1+/- mice it was missing or barely evident (Fig. 1D, E). In contrast, the other major connective tissue structure that is adjacent to the epithelium, the spiral limbus, maintained its normal size in Brn4-;Tbx1+/- mice (Fig. 1D, E).

The majority of the defects in Brn4- mice involved otic capsule and the spiral ligament, two structures known to express Brn4 during ear development. The Brn4 null phenotype has been previously described (Minowa et al. 1999; Phippard et al. 1999). However, our analysis uncovered many additional defects. In the basal turn of the cochlea, a conspicuous paucity of cells was sometimes evident within the spiral ligament of both Brn4- and Brn4-;Tbx1+/- mice (Fig. 1C). The thickness of the spiral ligament was reduced in the hook portion of the cochlea, which is the basal extremity of the ligament (Fig. 2B). Hypertrophy of the superior portion of the spiral ligament in the suprastrial region was also evident, and cells in the hypertrophied portion were less densely packed than in the remainder of the ligament. For example, the cochlea of a Brn4-;Tbx1+/- mouse exhibited suprastrial hypertrophy in which most of the scala vestibuli was filled with loose connective tissue (Fig. 2A). Melanocytes, normally found only within the stria vascularis and in the modiolus, were present in the hypertrophied spiral ligament, and Reissner's membrane was often attached at this site, distorting its normal shape (not shown).

Associated with a reduced spiral ligament was a missing stria vascularis (Fig. 2B). In other cases, the stria was missing in the lower basal turn without a thinning of the spiral ligament (as in Fig. 1B). In the upper basal turn, the portion of the spiral ligament to which the apical stria vascularis is normally attached was sometimes missing, resulting in the stria vascularis protruding away from the spiral ligament (Figs. 1B and 2B). Brn4 is not expressed in the stria, thus disruption of this structure is likely secondary to defects in the spiral ligament.

Finally, in contrast to the smooth outline of the bone surrounding the scala vestibuli in normal mice (Fig. 2C), Brn4- and Brn4-;Tbx1+/- mice displayed protrusions of bone that were frequently associated with an acellular mound of undefined eosinophilic substance (Fig. 2A, B). Furthermore, the bone covering the core of the cochlea (modiolus), which contains the auditory nerve fibers, was often incomplete (Fig. 2B). The composition of the cochlear bone appeared abnormal due to an increased volume of marrow within the medial portion of the bone (Fig. 1D-F).

\section{Abnormal spiral ligament fibrocytes in mutant mice}

Previous reports of Brn 4 null mice revealed alterations in the expression of key proteins in spiral ligament fibrocytes that likely contribute to the hearing loss found in these mutants (Minowa et al. 1999; Xia et al. 2002). Consistent with this, Brn4-;Tbx1+/- mice in this study were found to have profound hearing loss by auditory brain-stem response testing at 3 months of age (not shown). We hypothesized that Brn4-;Tbx1+/mice might display additional gene expression changes, and analyzed spiral ligament fibrocytes for expression of Aquaporin 1 (Aqp1), $\mathrm{Na}^{+}, \mathrm{K}^{+}$-ATPase and Connexin 26 (Cx26). There are four classes of cells within the spiral ligament that have characteristic locations and cytochemical profiles (Spicer and Schulte 1991). Type III fibrocytes are situated adjacent to the bony wall and express Aqp1 (Fig. 3A; Stankovic et al. 1995). In both Brn4- and Brn4-;Tbx+/- mice, AqpI positive cells were not confined to the lateral margin of the ligament, suggesting that they had either not migrated to their normal position or migrated away from that position (Fig. 3B; data not shown). Type II fibrocytes and marginal cells of the stria vascularis express $\mathrm{Na}^{+}, \mathrm{K}^{+}$-ATPase (Fig. 3C; Schulte and Adams 1989). In Brm4- and Brn4-;Tbx+/- mice, the stria stained normally for $\mathrm{Na}^{+}, \mathrm{K}^{+}$-ATPase, but type II fibrocytes stained faintly (Fig. 3D; data not shown). Furthermore, gap junctions are crucial for maintenance of cochlear fluid homeostasis, and Cx26 is expressed in all fibrocytes of the spiral ligament (Kikuchi et al. 1995). Expression of Cx26 in Brn4and Brn4-;Tbx1+/- mice was reduced to a scattering of cells situated near the bone and to basal and intermediate cells of the stria vascularis (Fig. 3E, F; data not shown). These data indicate that although no differences could be detected between the two genotypes, even portions of the cochlear connective tissue that exhibited normal histology had individual cell types that were cytochemically abnormal.

\section{Tbx1 and Brn4 are co-expressed during ear development}

Because both Tbx1 and Brn4 are expressed during embryogenesis, it is likely that the genetic interaction between these genes occurs during cochlear duct outgrowth. To confirm that both proteins are coexpressed in the periotic mesenchyme, we analyzed expression of Tbx1 and Brn 4 from E10.5 to E12.5 in the inner ear. These stages correspond to the time of 


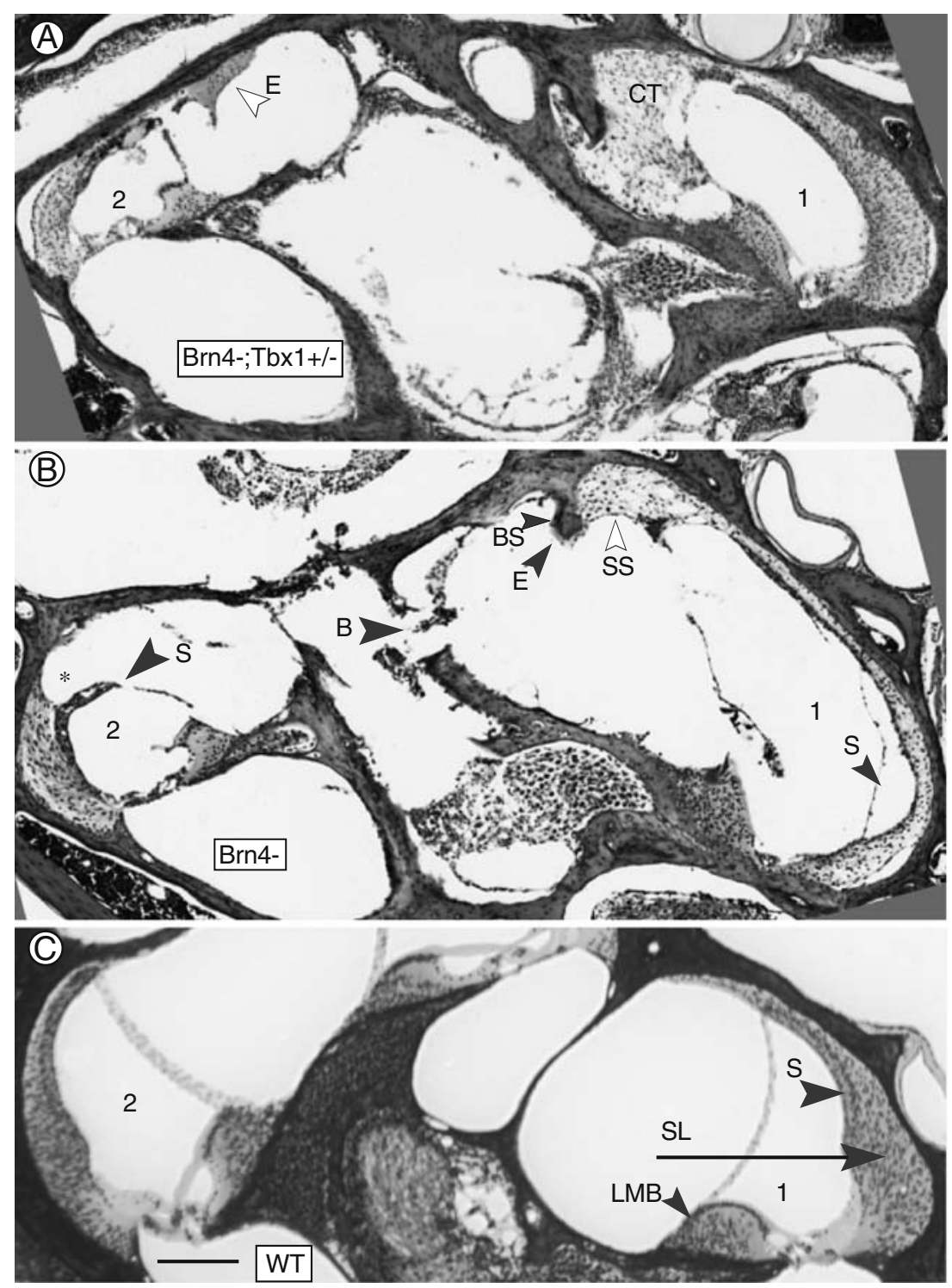

FIG. 2. Defects in the basal turn of mutant mice. A Basal turn of a Brn4-;Tbx1+/- mouse. Excessive connective tissue $(C T)$ has filled the space normally occupied by perilymph in the scala vestibuli. Abnormal eosinophilic material $(E)$ is present in the upper basal turn. B Basal turn of a Brn4- mouse. Hypertrophied suprastrial connective tissue (SS) is present in the lower basal turn, and the stria vascularis $(S)$ is missing. Note the adjacent spiral ligament is much thinner than in the corresponding area in $\mathbf{A}$ and $\mathbf{C}$. In the upper basal turn, the

cochlear duct formation and mesenchymal condensation around the otic vesicle. Expression of $T b x 1$ in the periotic mesenchyme was detected at E10.5 by immunohistochemistry on sagittal sections of wild type embryos. At this stage, Tbx 1 expression was confined to the ventral mesenchyme immediately adjacent to the otic vesicle (Fig. 4A). This expression continued at E11.5 in the medial half of the otic vesicle, with strongest expression in cells surrounding the forming cochlear duct (Fig. 4D). Along the medial-lateral axis, Tbx 1 expression in the periotic mesenchyme formed a spiral ligament is missing (asterisk) where it would normally be attached to the stria vascularis, and there are voids in the modiolar bone (B). C Basal turn of wild type cochlea showing normal morphology. (1) and (2) indicate lower basal and upper basal halfturns, respectively. $B$, missing bone; $B S$, bone spur; $C T$, connective tissue; $E$, eosinophillic substance; $L M B$, spiral limbus; $S L$, spiral ligament; $S$, stria vascularis; and $S S$, suprastrial region. The bar in C indicates $100 \mu \mathrm{m}$.

gradient that fell off laterally. Of note, this contrasts the expression domain of $T b x 1$ in the otic vesicle, which was expressed in a converse manner along the same axis (note absence of Tbx 1 expression in the otic epithelium in Fig. 4D and strong lateral expression in Fig. 4G). By E12.5, expression of Tbx1 in the periotic mesenchyme was restricted to the mesenchymal cells surrounding the most medial portion of the cochlear duct (Fig. 4G).

$B r n 4$ is expressed in the hindbrain and neural tube as early as E9.5; however, expression in the periotic 


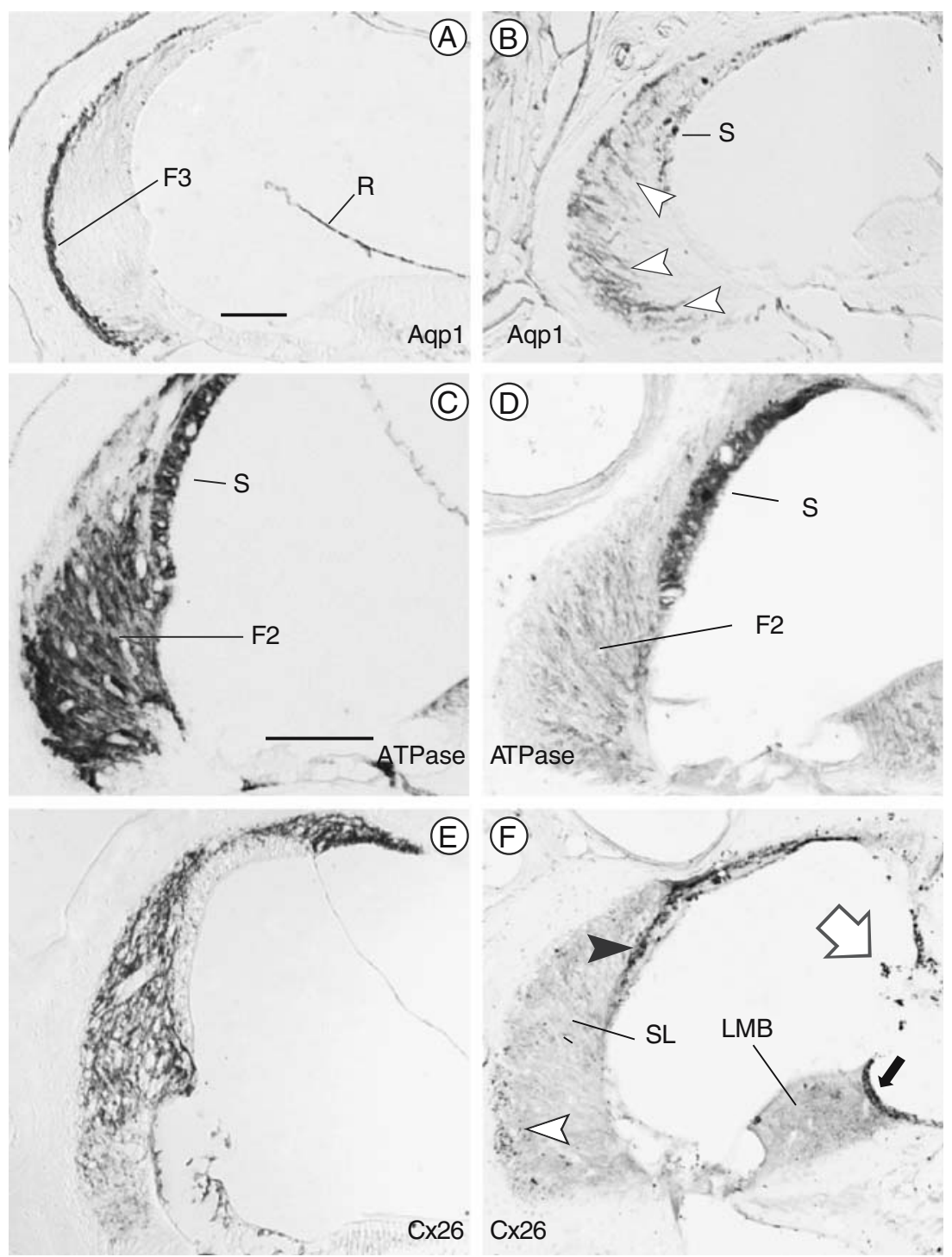

FIG. 3. Spiral ligament fibrocytes are abnormal in mutant mice. Immunostaining of wild type (A, C, and $\mathbf{E})$ and Brn4-;Tbx $1+/-(\mathbf{B}$, $\mathbf{D}$, and $\mathbf{F}$ ) inner ears. A Normal distribution of type III fibrocytes (F3) at the junction of the spiral ligament and the petrous bone immunostained for Aquaporin 1 (Aqp1). B Aqp1 immunostained cells in Brn4-;Tbx1+/- ears are situated broadly throughout the spiral ligament (arrowheads). C Type II fibrocytes (F2) in the spiral ligament express $\mathrm{Na}^{+}, \mathrm{K}^{+}$-ATPase in wild type inner ears. The stria vascularis $(S)$ also exhibits positive immunostaining. D The type II fibrocytes in $\mathrm{Brn} 4-; \mathrm{Tbx} 1+/-$ mice only weakly express $\mathrm{Na}^{+}, \mathrm{K}^{+}-$ ATPase; however, the stria remains strongly positive. E Connexin 26

mesenchyme was not detected until E10.5 (Fig. 4B). Coexpression of Brn4 and Tbx 1 occurred in the posterior and ventral periotic mesenchyme in the cells surrounding the site of the future cochlear duct. In Tbx 1-/- embryos, Brn4 expression was absent or reduced in the same region, suggesting either a downregulation of Brn4 expression or loss of cells (Fig. 4C). At E11.5, Brn4 expression expanded to most periotic mesenchyme cells immediately surrounding the otic vesicle. In a medial section though
(Cx26) is expressed broadly in the spiral ligament of a wild type inner ear. F Cx26 immunostaining of the inner ear of a Brn4-;Tbx1+/mouse shows only a few positive cells within the spiral ligament (open arrowhead), but strongly stains the strial basal and intermediate cells (closed arrowhead). The spiral limbus $(L M B)$ expresses $C \times 26$ in wild type mice (not shown); however, it fails to stain in Brn4-;Tbx $1+/-$ mice save for a dark strip of supralimbal cells (closed arrow). Melanocytes stain nonspecifically (open arrow). F2, type II fibrocytes; F3, type III fibrocytes; $R$, Reissner's membrane; $S$, stria vascularis; $S L$, spiral ligament; and $L M B$, spiral limbus. Scale bar is $100 \mu \mathrm{m}$.

the developing cochlear duct, Brn4 expression was strongest in the ventral and posterior mesenchyme (Fig. 4E) and was lost in the same region in Tbx1-/embryos (Fig. 4F). By E12.5, Brn4 was expressed throughout the periotic mesenchyme (Fig. 4H), and colocalized with $T b x 1$ in mesenchymal cells that condense around cochlear duct epithelium. Expression of Tbxl was unchanged in Brn4-/- embryos, indicating that $T b x 1$ may lie upstream of $B r n 4$ (not shown). 

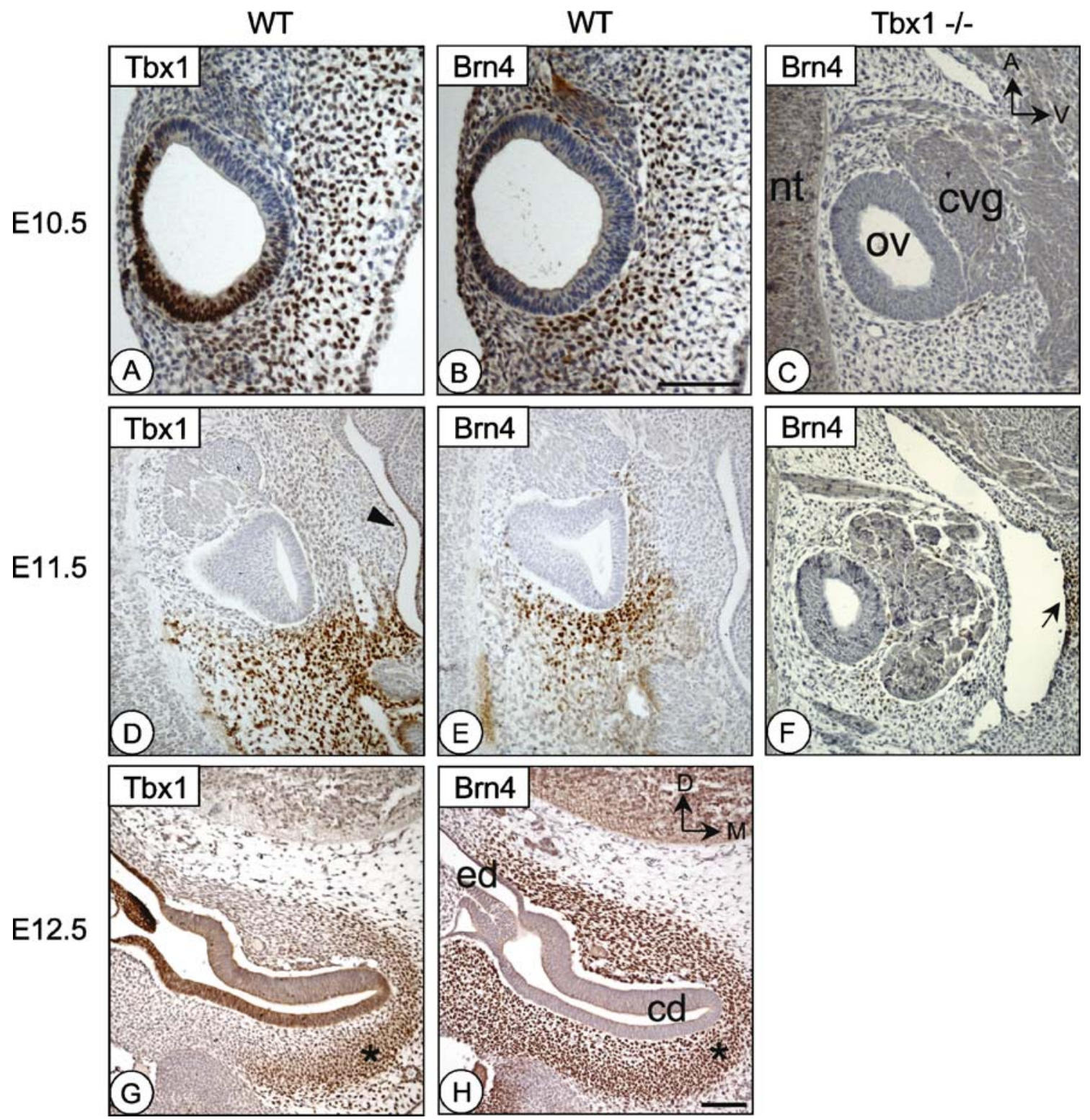

FIG. 4. Tbx 1 and Brn4 are coexpressed in the periotic mesenchyme during development. Adjacent sections of the inner ear at E10.5 (A and B), E11.5 (D and E), and E12.5 (G and $\mathbf{H})$ immunostained for either Tbx1 (A, D, and $\mathbf{G}$ ) or Brn4 (B, E, and $\mathbf{H})$ protein. $\mathbf{C}$ and $\mathbf{F}$ are stage matched sections of Tbx1-/- embryos stained for Brn4. A-F are sagittal sections, while $\mathbf{G}$ and $\mathbf{H}$ are transverse. At E10.5, both Tbx1 (A) and Brn4 (B) are expressed in the ventral and posterior periotic mesenchyme surrounding the otic vesicle. Tbx 1 is also expressed in the posterior-dorsal otic vesicle epithelium. At E11.5 in medial sections through the otic vesicle, Tbx 1 (D) and Brn4 (E) are coexpressed in the posterior mesenchyme. Tbx1 expression can also be detected in the pharyngeal endoderm (arrowhead in D). Tbx1-/-

Cell survival is abnormal in Brn4-;

Tbx 1+/- embryos

We hypothesized that the shortened cochlea observed in Brn4-;Tbx1+/- mice may be due to defects in cell survival in the cochlear epithelium during outgrowth embryos (which have an enlarged ganglion), show absent or strongly reduced Brn4 expression in the periotic mesenchyme at E10.5 (C) or E11.5 (F). Brn4 expression in the neural tube $(n t)$ and the second pharyngeal arch mesenchyme remains (arrow in $\boldsymbol{F}$ ). At E12.5, Tbx 1 expression is confined to only the most medial region of the periotic mesenchyme $(\mathbf{G})$, while Brn4 is expressed in a much broader cell population $(\mathbf{H})$. An asterisk marks the area of coexpression in $(\mathbf{G})$ and (H). cd, cochlear duct; $c v g$, cochleovestibular ganglion; ed, endolymphatic duct; $n t$, neural tube; and $o v$, otic vesicle. Orientation shown in $\mathbf{C}$ applies to $\mathbf{A}-\mathbf{F}$, while orientation in $\mathbf{H}$ applies to $\mathbf{G}$ and $\mathbf{H}$. Scale bar in $\boldsymbol{H}$ is $200 \mu \mathrm{m}$ and applies to $\mathbf{C}-\mathbf{H}$. Scale bar in $\boldsymbol{B}$ is $200 \mu \mathrm{m}$ and applies to $\mathbf{A}$ and $\mathbf{B}$.

of the cochlear duct. Expression of $T b x 1$ was recently shown to be required for this process, as conditional ablation of Tbx 1 in the periotic mesenchyme led to a decrease in proliferation of both periotic mesenchyme cells and a subset of cochlear epithelial cells (Xu et al. 2007a). Since Brn4 and Tbx1 are coex- 

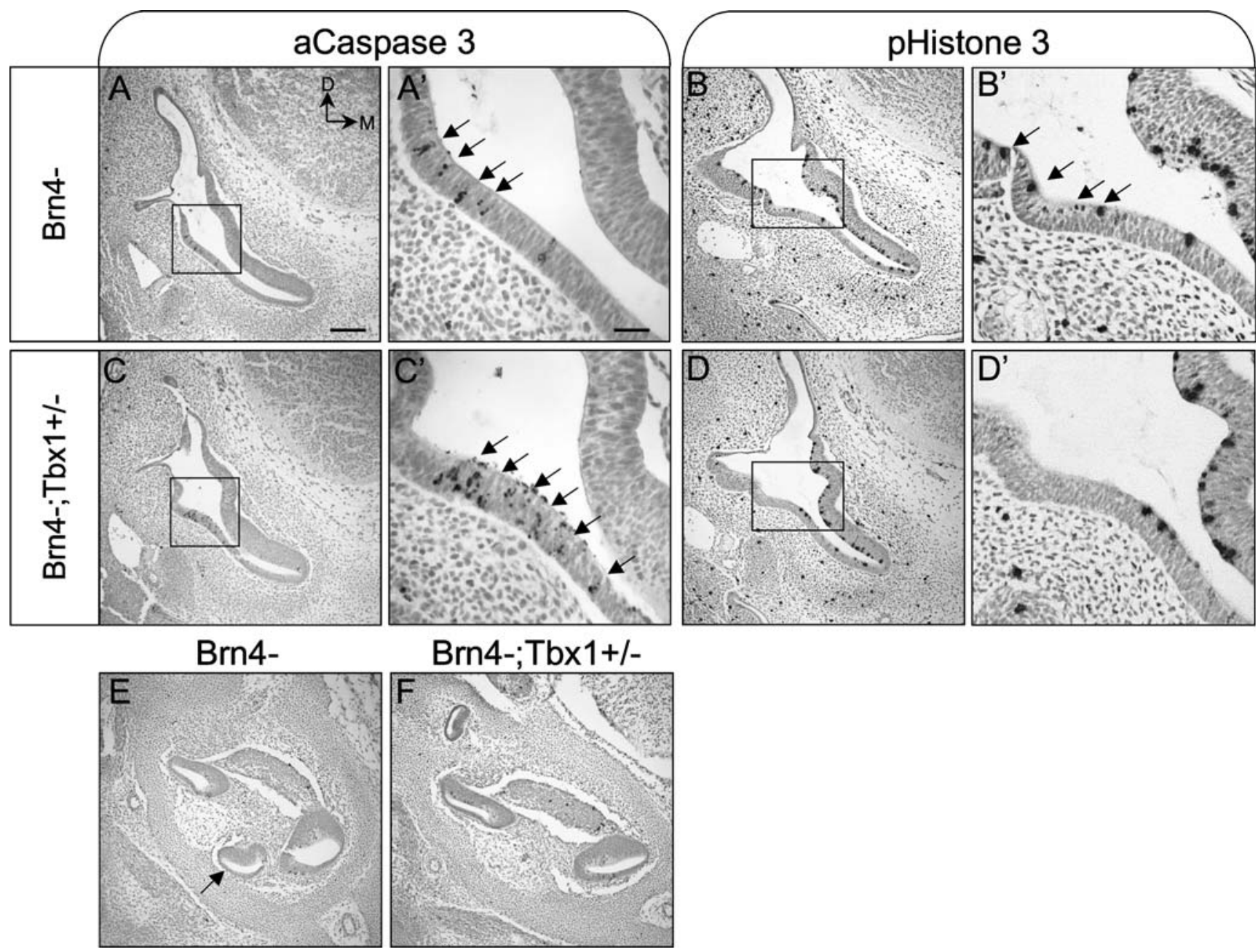

Brn4-;Tbx1+/-

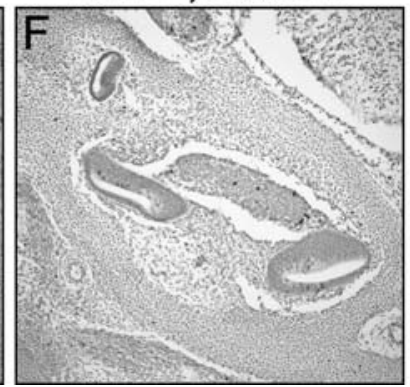

FIG. 5. Cell survival is abnormal in the basal cochlea of Brn4-; Tbx $1+/-$ embryos. Transverse sections through the otic vesicle of E12.5 Brn4- (A and B) and Brn4-;Tbx1+/- (C and D) embryos stained for the apoptosis marker activated Caspase 3 (aC3; A, $\mathbf{A}^{\prime}, \mathbf{C}$, and $\left.\mathbf{C}^{\prime}\right)$ or the mitotic marker phospho-Histone $3\left(\mathrm{pH} 3 ; \mathbf{B}, \mathbf{B}^{\prime}, \mathbf{D}\right.$, and $\mathbf{D}^{\prime}$ ). $\mathbf{A}$ and $\mathbf{A}^{\prime}$ At E12.5, apoptotic cells are present in the lateral wall of Brn4- embryos (arrows). A magnified view of the boxed region is shown in $\mathbf{A}^{\prime}$. B and $\mathbf{B}^{\prime}$ Proliferating cells are detected throughout

pressed in the periotic mesenchyme from E10.5 to E12.5, we analyzed embryos at E12.5 for abnormal apoptosis and proliferation in the inner ear. Apoptosis was detected using an antibody to activated Caspase 3, while cell proliferation was detected using an antibody to the M-phase marker phospho-Histone H3. At this stage, Brn4- embryos ( $n=4)$ exhibited regional apoptosis in the lateral wall of the basal cochlea, similar to what has been reported in WT embryos (Lang et al. 2000; Nishikori et al. 1999) (Fig. 5A). In this same region, the number of apoptotic cells was increased in Brn4-;Tbx $1+/-$ embryos ( $n=4)$ (Fig. 5C). Embryos in which $T b x 1$ has been conditionally ablated in the periotic mesenchyme display increased apoptosis in this region as well (Xu et al. 2007a). We next analyzed cochlear duct in Brn4- embryos. C and $\mathbf{C}^{\prime}$ Brn4-;Tbx1+/- embryos display increased apoptosis in the lateral wall of the cochlea, and a reduced proliferation in this same region $\left(\mathbf{D}\right.$ and $\left.\mathbf{D}^{\prime}\right)$. $\mathbf{E}$ and $\mathbf{F}$ While some dying cells were detected, no significant differences in apoptosis were seen in the cochlear duct or periotic mesenchyme between Brn4- and Brn4-;Tbx1+/- embryos; however, the apical cochlea (arrow in $\boldsymbol{E}$ ) is missing in Brn4-;Tbx+/- embryos. Scale bar indicates $200 \mu \mathrm{m}$ in $\mathbf{A}-\mathbf{F}$ and $50 \mu \mathrm{m}$ in $\mathbf{A}^{\prime}-\mathbf{D}^{\prime}$.

embryos for a decrease in cell proliferation at E12.5 $(n=4)$. While no differences in the number of proliferating cells could be detected over the entire cochlea between Brm4- and Brm4-;Tbx1+/- embryos, Brn4-; Tbx $1+/-$ mutants did display a localized reduction in proliferation in the lateral wall of the cochlea (Fig. 5B, $\mathrm{D})$. This corresponds to the region where an increase apoptotic cells were detected (compare Fig. $5 \mathrm{C}^{\prime}$ to $\mathrm{D}^{\prime}$ ).

We also examined cell survival in mutant embryos at E14.5 $(n=3)$. No differences in apoptosis could be detected at this stage. However, the most apical portion of the cochlear duct was missing in Brn4-; Tbx1+/- embryos, indicating that changes in cell survival likely take place before this stage during inner ear development (Fig. 5E,F). 


\section{DISCUSSION}

Tbx 1 and Brn 4 genetically interact in the periotic mesenchyme

Mondini dysplasia is a common inner ear malformation in individuals affected with congenital deafness; however, its etiology is largely unknown (Zheng et al. 2002). This type of defect is found in association with several genetic syndromes, including VCFS/DGS resulting from 22q11.2 deletions or mutations in TBX1 and in patients with DFN3 due to mutations in $B R N 4$. To further understand the roles of $T B X 1$ and $B R N 4$ in inner ear development, we have examined mouse models with null alleles in both genes. $T b x 1$ is expressed in the otic vesicle and the periotic mesenchyme, and it has been shown that both domains of expression are required for inner ear development. Lack of $T b x 1$ in the otic vesicle results in failed development of a vestibular system and cochlea (Arnold et al. 2006a), while lack of Tbxl in the periotic mesenchyme results in failed cochlear outgrowth (Xu et al. 2007b). It is interesting to note that while Brm4 expression is reduced in Tbx 1 null mice, it is unaffected in conditional mutants where $T b x 1$ is ablated in the otic vesicle (Arnold et al. 2006a). These data led us to investigate a possible genetic interaction between Brn 4 and Tbx1.

In this study, the importance of the periotic mesenchyme, and specifically Tbx1 and Brn4, in cochlear development has been established by performing genetic intercrosses. We show that Tbx1 and Brn4 cooperate to promote cochlear coiling. Compared to Brn4- mice, Brn4-;Tbx1+/- mutants exhibited a reduction from the normal four half turns of the cochlea to two or three. This phenotype displayed complete penetrance, in contrast to the milder and incompletely penetrant phenotype of Brn4- mice. In addition to a shortened cochlea, Brn4-;Tbx1+/- mice displayed an absent interscalar septum. Importantly, these defects resemble Mondini dysplasia found in patients with VCFS/DGS and DFN3. We suggest that periotic mesenchyme-mediated signals that direct the upward spiraling growth of the cochlea are aberrant in Brn4-;Tbx1+/- mutants, making them an excellent model for studying the molecular pathogenesis of Mondini dysplasia.

We hypothesize that $T b x 1$ functions together with $\mathrm{Brn} 4$ to modulate gene expression in a critical subset of periotic mesenchyme cells on the ventral border of the otic vesicle, and that interactions between these cells and the otic vesicle epithelium are necessary for proper cochlea formation and cell survival. Multiple signaling pathways are known to play a role in epithelial-mesenchymal interactions during ear development, including Shh signaling (Liu et al. 2002). In Shh null mutants, which lack a cochlea, both $T b x 1$ and $\mathrm{Brn} 4$ expression in the periotic mesenchyme are lost (Riccomagno et al. 2002). Further, Shh signaling was recently shown to mediate outgrowth of the distal cochlear duct via activation of Gli transcription factors expressed in the periotic mesenchyme and otic vesicle (Bok et al. 2007). The cochlear defects seen in the mutants analyzed in this study indicate that Tbx1 and Brn 4 may be targets of Gli activity. Still unidentified, however, is the signaling pathway downstream of $T b \times 1$ and $B m 4$ that is required for cochlear morphogenesis.

\section{Role of TBX1 and BRN4 in human disease}

We found Brm4- and Brn4-;Tbx1+/- mice to be deaf and display abnormalities in structures derived from the periotic mesenchyme. The defects in the lateral wall of the cochlea provide an explanation for the deafness found in Brn 4 null mice despite little evidence of physical disruption of the organ of Corti. Dysmorphologies of the spiral ligament and stria vascularis could account for lack of normal function, but even in sites where the morphology was normal, immunostaining showed that the cytochemistry of the cells was abnormal. The reduction of Cx26 expression among spiral ligament fibrocytes indicates the lack of a gap junction pathway for recycling of $\mathrm{K}^{+}$ions from perilymph to the stria vascularis. The principal site of $\mathrm{K}^{+}$uptake from perilymph is though to be type II fibrocytes (Kikuchi et al. 1995; Kikuchi et al. 2000). Unlike strial marginal cells, which take up $\mathrm{K}^{+}$basolaterally and expel it through their apical membranes, type II fibrocytes are not epithelial and have no apical membrane through which to expel ions. Without a route of egress of accumulated $\mathrm{K}^{+}$, the type II cells must desist from accumulating ions, which appears to be accomplished by down regulating $\mathrm{Na}^{+}, \mathrm{K}^{+}$-ATPase. The disruption in $\mathrm{K}^{+}$homeostasis is likely the cause of sensorineural deafness in the resulting mutant mice due to both a loss of endolymphatic potential and a lack of $\mathrm{K}^{+}$ions to carry receptor currents.

While the majority of the defects in Brn4- mice support a cell-autonomous role for $\mathrm{Br} 4$ in the periotic mesenchyme, the reduction in the number of cochlear turns in a subset of these mice indicates a role for $\mathrm{Brm} 4$ in mesenchymal signaling to the otic vesicle (this study and Phippard et al. 1999). However, penetrance of this defect may depend in part on the particular mutant allele and genetic background. We found that $85 \%$ of Brn4- mice exhibited the normal number of cochlear turns, while $15 \%$ were found to display three to four half turns. Variation in nucleotide sequence of specific genes, such as $T b x 1$, may account for genetic background differences and might serve as genetic modifiers of Brn4. Based upon our work, it is possible that variation in TBX1 expression levels resulting from 
nucleotide polymorphisms might contribute to the incidence of pathologies such as Mondini dysplasia in cases of DFN3. Alternatively, polymorphisms in BRN4 could affect the risk of Mondini dysplasia in VCFS/ DGS patients that are hemizygously deleted for TBX1.

\section{ACKNOWLEDGEMENTS}

We thank Dr. Vimla Aggarwal and Dr. Jun Liao for their critical reading of the manuscript and intellectual advice. This work is supported by the National Institutes of Health (DC05186-03) to B.E.M and P30 DC005209 to J.C.A. E.M.B is supported by the Ruth L. Kirshstein National Research Service Award (DC008239-02).

\section{REFERENCES}

Arellano B, Ramirez Camacho R, Garcia Berrocal JR, Villamar M, Del Castillo I, Moreno F. Sensorineural hearing loss and Mondini dysplasia caused by a deletion at locus DFN3. Arch. Otolaryngol. Head Neck Surg. 126:1065-1069, 2000.

Arnold JS, Braunstein EM, Ohyama T, Groves AK, Adams JC, Brown MC, Morrow BE. Tissue-specific roles of Tbx1 in the development of the outer, middle and inner ear, defective in 22q11DS patients. Hum. Mol. Genet. 15:1629-1639, 2006 a.

Arnold JS, Werling U, Braunstein EM, Liao J, Nowotschin S, Edelmann W, Hebert JM, Morrow BE. Inactivation of Tbx1 in the pharyngeal endoderm results in 22q11DS malformations. Development 133:977-987, 2006 b.

Bok J, Dolson DK, Hill P, Ruther U, Epstein DJ, Wu DK. Opposing gradients of Gli repressor and activators mediate Shh signaling along the dorsoventral axis of the inner ear. Development 134:1713-1722, 2007.

Chang W, ten Dijke P, Wu DK. BMP pathways are involved in otic capsule formation and epithelial-mesenchymal signaling in the developing chicken inner ear. Dev. Biol. 251:380-394, 2002.

de Kok YJ, van der Maarel SM, Bitner-Glindzicz M, Huber I, Monaco AP, Malcolm S, Pembrey ME, Ropers HH, Cremers FP. Association between X-linked mixed deafness and mutations in the POU domain gene POU3F4. Science 267:685-688, 1995.

Digilio MC, Pacifico C, Tieri L, Marino B, Giannotti A, Dallapiccola B. Audiological findings in patients with microdeletion 22q11 (di George/velocardiofacial syndrome). Br. J. Audiol. 33:329-333, 1999.

Ford LC, Sulprizio SL, Rasgon BM. Otolaryngological manifestations of velocardiofacial syndrome: a retrospective review of 35 patients. Laryngoscope 110:362-367, 2000.

Holden PK, Linthicum FH, JR. Mondini dysplasia of the bony and membranous labyrinth. Otol. Neurotol. 26:133, 2005.

Kikuchi T, Kimura RS, Paul DL, Adams JC. Gap junctions in the rat cochlea: immunohistochemical and ultrastructural analysis. Anat. Embryol. (Berl) 191:101-118, 1995.

Kikuchi T, Kimura RS, Paul DL, Takasaka T, Adams JC. Gap junction systems in the mammalian cochlea. Brain Res. Brain Res. Rev. 32:163-166, 2000.

Lang H, Bever MM, Fekete DM. Cell proliferation and cell death in the developing chick inner ear: spatial and temporal patterns. J. Comp. Neurol. 417:205-220, 2000.
Liao J, Kochilas L, Nowotschin S, Arnold JS, Aggarwal VS, Epstein JA, Brown MC, Adams J, Morrow BE. Full spectrum of malformations in velo-cardio-facial syndrome/DiGeorge syndrome mouse models by altering Tbx1 dosage. Hum. Mol. Genet. 13:1577-1585, 2004.

Liu W, Li G, Chien JS, Raft S, Zhang H, Chiang C, Frenz DA. Sonic hedgehog regulates otic capsule chondrogenesis and inner ear development in the mouse embryo. Dev. Biol. 248:240-250, 2002.

Minowa O, Ikeda K, Sugitani Y, Oshima T, Nakai S, Katori Y, Suzuki M, Furukawa M, Kawase T, Zheng Y, Ogura M, Asada Y, Watanabe K, Yamanaka H, Gotoh S, Nishi-Takeshima M, Sugimoto T, Kikuchi T, TAKasaka T, NodA T. Altered cochlear fibrocytes in a mouse model of DFN3 nonsyndromic deafness. Science 285:1408-1411, 1999.

Nishikori T, Hatta T, KaWAuchi H, Otani H. Apoptosis during inner ear development in human and mouse embryos: an analysis by computer-assisted three-dimensional reconstruction. Anat. Embryol. (Berl) 200:19-26, 1999.

Ohtani I, Schuknecht HF. Temporal bone pathology in DiGeorge's syndrome. Ann. Otol. Rhinol. Laryngol. 93:220-224, 1984.

Phelps PD, Reardon W, Pembrey M, Bellman S, Luxom L. X-linked deafness, stapes gushers and a distinctive defect of the inner ear. Neuroradiology 33:326-320, 1991.

Phippard D, Heydemann A, Lechner M, Lu L, Lee D, Kyin T, Crenshaw EB, 3RD. Changes in the subcellular localization of the Brn4 gene product precede mesenchymal remodeling of the otic capsule. Hear. Res. 120:77-85, 1998.

Phippard D, Lu L, Lee D, Saunders JC, Crenshaw EB, 3Rd. Targeted mutagenesis of the POU-domain gene Brn4/Pou3f4 causes developmental defects in the inner ear. J. Neurosci. 19:5980-5989, 1999.

Pirvola U, Zhang X, Mantela J, Ornitz DM, Ylikoski J. Fgf9 signaling regulates inner ear morphogenesis through epithelial-mesenchymal interactions. Dev Biol 273:350-360, 2004.

Raft S, Nowotschin S, Liao J, Morrow BE. Suppression of neural fate and control of inner ear morphogenesis by Tbx1. Development 131:1801-1812, 2004.

Reyes MR, LeBlanc EM, Bassila MK. Hearing loss and otitis media in velo-cardio-facial syndrome. Int. J. Pediatr. Otorhinolaryngol. 47: 227-233, 1999.

Riccomagno MM, Martinu L, Mulheisen M, Wu DK, Epstein DJ. Specification of the mammalian cochlea is dependent on Sonic hedgehog. Genes Dev. 16:2365-2378, 2002.

Schulte BA, Adams JC. Distribution of immunoreactive Na+,K+-ATPase in gerbil cochlea. J. Histochem. Cytochem 37:127-134, 1989.

SPICER SS, SCHUlTE BA. Differentiation of inner ear fibrocytes according to their ion transport related activity. Hear. Res. 56:53-64, 1991.

Stankovic KM, AdAms JC, Brown D. Immunolocalization of aquaporin CHIP in the guinea pig inner ear. Am. J. Physiol. 269: C1450-C1456, 1995.

Vitelli F, Viola A, Morishima M, Pramparo T, Baldini A, Lindsay E. TBX1 is required for inner ear morphogenesis. Hum. Mol. Genet. 12:2041-2048, 2003.

Xia AP, Kikuchi T, Minowa O, Katori Y, Oshima T, Noda T, Ineda K. Late-onset hearing loss in a mouse model of DFN3 nonsyndromic deafness: morphologic and immunohistochemical analyses. Hear. Res 166:150-158, 2002.

Xu H, Chen L, Baldini A. In vivo genetic ablation of the periotic mesoderm affects cell proliferation, survival and differentiation in the cochlea. Dev. Biol. 310(2):329-340, 2007, Oct 15.

Xu H, Viola A, Zhang Z, Gerken CP, Lindsay-Illingworth EA, Baldini A. Tbx1 regulates population, proliferation and cell fate determination of otic epithelial cells. Dev. Biol. 302:670-682, 2007b.

Zheng Y, Schachern PA, Cureoglu S, Mutlu C, Dijalilian H, Paparella MM. The shortened cochlea: its classification and histopathologic features. Int. J. Pediatr. Otorhinolaryngol. 63:29-39, 2002. 